\title{
Adaptive Antenna-Activation Based Beamforming for Large-Scale MIMO Communication Systems of High Speed Railway
}

\author{
Meng Cheng ${ }^{1,2,3}$, Shaoshi Yang ${ }^{3}$, Xuming Fang*2 \\ ${ }^{1}$ Post-Doctoral Working Station, Wuhan Railway Bureau, Wuhan 430070, Hubei Province, China \\ ${ }^{2}$ Provincial Key Laboratory of Information Coding and Transmission, Southwest Jiaotong University, \\ Chengdu 610031, Sichuan Province, China \\ ${ }^{3}$ School of Electronics and Computer Science, University of Southampton, Southampton, SO17 1BJ, UK
}

\begin{abstract}
Large-scale array aided beamforming improves the spectral efficiency (SE) as a benefit of high angular resolution. When dual-beam downlink beamforming is applied to the train moving towards cell edge, the inter-beam ambiguity (IBA) increases as the directional difference between beams becomes smaller. An adaptive antenna activation based beamforming scheme was proposed to mitigate IBA. In the district near the base station (BS), all antenna elements (AEs) were activated to generate two beams. As the distance from the train to the BS increased, only the minimum number of AEs satisfying the resolution criterion would be activated. At the cell edge, one beam was switched off due to intolerable IBA. The proposed scheme can achieve SE gain to the non-adaptive scheme and show more robustness against the direction-of-arrival (DOA) estimation error.
\end{abstract}

Keywords: wireless communications; adaptive antenna activation; large-scale antenna array; downlink beamforming; high speed railway

\section{INTRODUCTION}

Passenger-oriented communication systems (POCS), which are capable of providing high-reliability and high-rate services for high speed railway (HSR) passengers has drawn increasing research interests in recent years [1]-[12]. This system faces several challenges imposed by a high Doppler shift, by the estimation of rapidly time-varying channels and frequent handovers. Nevertheless, it also enjoys inherent advantages, such as a predictable vehicle speed and the location information provided by the dedicated communication based train control (CBTC) subsystem [13].

Several spatial multiplexing, diversity and beamforming based schemes have been conceived for small-scale multiple-antenna aided POCS systems of HSR [9]-[12]. To be more specific, an efficient handover scheme was proposed in [10] for exploiting the benefits of beamforming and of the positioning information. In [11], a location-information assisted opportunistic beamforming scheme was proposed for the multi-user long-term-evolution (LTE) system employed in HSR. The beamforming technique was also combined with space-time block coding for the case of achieving diversity gain in POCSs [12]. As pointed out in [9], due to the rapidly varying DOA caused by high mobility, it is impractical to mechanically steer a pencil beam for tracking the antenna on the train. From the 
This paper proposed an AAA based beamforming scheme for LS-MIMO aided communication systems of HSR scenario, which activates different number of BS AEs and generates distinct number of serving beams in different district track-side downlink transmitter, the beam can only be generated by adjusting the weighting factors of the array. However, when smallscale MIMO techniques are invoked in POCS, it remains a challenge to generate sufficiently narrow beams due to their inaccurate angular resolution; hence the onboard receiver experiences a strong line-of-sight (LOS) path in most HSR scenarios, which limits the attainable performance. As a result, the benefits of beamforming remain limited in small-scale MIMO systems.

As a remedy, the emerging large-scale MIMO (LS-MIMO) techniques might be invoked but they impose further design challenges, especially in the context of the POCS of HSR. On the one hand, the more AEs the $\mathrm{BS}$ uses, the higher the angular resolution becomes [14]. Furthermore, multi-user MIMO techniques can be employed for achieving an increased multiplexing gain even in strong LOS scenarios [15], provided that the number of BS AEs is higher than the number of single-antenna users [16]. It was predicted in [15] that LS-MIMO aided POCS are capable of serving as many as 42 mobile stations (MS) at a velocity of 320 to $574 \mathrm{~km} / \mathrm{h}$ in each cell onboard the French Train a Grande Vitesse (TGV), whilst maintaining the $\mathrm{SE}$ as $36.5 \mathrm{bps/}$ $\mathrm{Hz}$ under the idealized simplifying assumption of having an unlimited number of BS AEs. On the other hand, when characterizing the performance of transmit beamforming/precoding (TBF/TPC) in LS-MIMO communication systems, it was suggested to take the angular power spectrum into consideration, because the effect of the far-field scattering environment is significant in LS-MIMO systems [17].

In addition to $\mathrm{TBF}$, there are also several generic TPC algorithms [18]-[22]. In [18], a low-complexity zero-forcing (ZF) aided TPC scheme was proposed for achieving the same performance as eigenbeamforming, while requiring an order of magnitude fewer AEs. Both the ZF and the minimum mean squared error (MMSE) based precoding schemes were shown to reach $98 \%$ of the dirty-paper coding capacity with as few as 20 AEs at the BS in a residential-area [19]. Furthermore, as the number of BS AEs tends to infinity, both the effects of Gaussian noise and of co-channel interference are substantially reduced, but the influence of pilot contamination which refers to the interference which results from unavoidable re-use of uplink pilot sequences by terminals in different cells still exists [20]. As a remedy, the authors of [21] combined the linear TPC based on the fast-fading coefficients with another TPC design based on both the path-loss and shadowing for eliminating the inter-cell interference. For the uplink, the classic matched filter (MF) approaches the performance of the optimal maximum-likelihood detector, when the number of BS AEs is larger than the total number of MS transmit antennas, whilst the MMSE detector can support more users than the MF, when the pilot contamination is not excessive [22].

Both single and multi-beam beamforming modes were analyzed in [23], where the optimal number of beams was calculated under the intention of maximizing the users' sumrate by reducing the IBA. Both optimal singleand multi-beam TBF were proposed in [24] under per-antenna power constraints. In [25] the users were grouped according to the pathloss and each group was assigned a specific beamforming scheme [26], which was demonstrated to achieve the same performance as the idealized scenario relying on hundreds of BS AEs, despite a 10-fold reduction in the number of AEs, e.g. from three hundred to thirty. In [15] and [27], the users were divided into different groups according to their speed. Despite significant progress, most exciting contributions [15]-[27] focus on conventional cellular systems, where the MSs roam at a low speed and have compact dimensions.

By contrast, in this paper the performance of dual-beam beamforming is analyzed in LS-MIMO aided HSR scenarios and a novel adaptive antenna-activation (AAA) based beamforming scheme is proposed for HSR scenarios. The proposed scheme exploits the high angular resolution of LS-MIMO for simultaneously achieving high array gain and 
multiplexing gain.

The rest of this paper is organized as follows. In Section II we describe the application scenario considered, while Section III analyzes the dual-beam beamforming technique, which is then extended to the proposed AAA based beamforming scheme. Finally, in Section IV, the performance of these BF schemes is characterized, while Section V concludes the paper and suggests some future work.

\section{TOPOLOGY OF HSR COMMUNiCATION SYSTEMS}

We consider a linear topology for the LS-MIMO aided HSR system of Fig. 1. The trackside BS is equipped with a uniform linear array (ULA) composed of hundreds of AEs, and its coverage radius is $R$. The reference $\mathrm{AE}$ (e.g. the first AE) of the BS is denoted as $O$ which projects to the ground as $O^{\prime}$ as seen in Fig. 1. The antennas of the mobile relay station (MRS), which relays the signals to the passengers, on the train are denoted as $A$ and $B$, which are sufficiently far apart from each other (e.g. at the front and rear of the train, respectively). Their projecting points on the ground are $A^{\prime}$ and $B^{\prime}$. The perpendicular distance from $O^{\prime}$ to the line determined by $A^{\prime}$ ' and $B^{\prime}$ is $d_{\text {min }}$. Still considering Fig. 1, the length of $O O^{\prime}$ is $h_{B S}$ (i.e. the height of the BS), while the length of $A A^{\prime}$ ' is $h_{M R S}$ (i.e. the height of the MRS). The length of $A B$, i.e., the length of the train, is $l_{T}$, while the distance $O^{\prime} A$ ' is denoted as $s$, which can be obtained with the aid of the predicted location information. The angle between $O^{\prime} A^{\prime}$ and $O^{\prime} x$ in Fig. 1 is $\phi_{1}$, while that between $O^{\prime} B^{\prime}$ and $O^{\prime} x$ is $\phi_{2}$.

It is assumed that the AE separation of the BS is $\lambda \Delta$ and the number of active BS AEs is $N$, where $\lambda$ is the wavelength and $\Delta$ is the normalized receive AE separation, normalized to the wavelength [14, Chapter 7.2]. More explicitly, when all the AEs of the BS are activated, we have $N=N_{\max }$. Then, the normalized length of the ULA is expressed as $l_{N}=N \Delta$. To satisfy the angular resolution requirement [14], the difference between $\cos \phi_{1}$ and $\cos \phi_{2}$ should be larger than $1 / l_{N}$, i.e.,

$$
\left|\cos \phi_{1}-\cos \phi_{2}\right| \geqslant \frac{1}{l_{N}},
$$

which guarantees that the signals transmitted to $A$ and $B$ can be distinguished by the receive antenna. Upon satisfying (1), from Fig. 1, we have:

$\left|\frac{\sqrt{s^{2}-d_{\min }^{2}}}{s}-\frac{\sqrt{s^{2}-d_{\min }^{2}}-l_{T}}{\sqrt{d_{\min }^{2}+\left(\sqrt{s^{2}-d_{\min }^{2}}-l_{T}\right)^{2}}}\right| \geqslant \frac{1}{N \Delta}$

As $s$ changes smoothly from $d_{\text {min }}$ to $R$ when the train moves from the cell center to the cell edge, the minimum number $N_{\min }$ of the BS AEs to be activated at location $s$ according to the angular resolution criterion can be calculated as follows:

$N_{\min }=\left|\frac{1}{\Delta\left|\frac{\sqrt{s^{2}-d_{\min }^{2}}}{s}-\frac{\sqrt{s^{2}-d_{\min }^{2}}-l_{T}}{\sqrt{d_{\min }^{2}+\left(\sqrt{s^{2}-d_{\min }^{2}}-l_{T}\right)^{2}}}\right|}\right|$

\section{BEAMFORMING SCHEMES FOR LS- MiMO COMMUNICATION SYSTEMS OF HSR}

\subsection{Dual-beam beamforming technique in HSR}

Before the emergence of the LS-MIMO concept, it was impractical to implement the du-

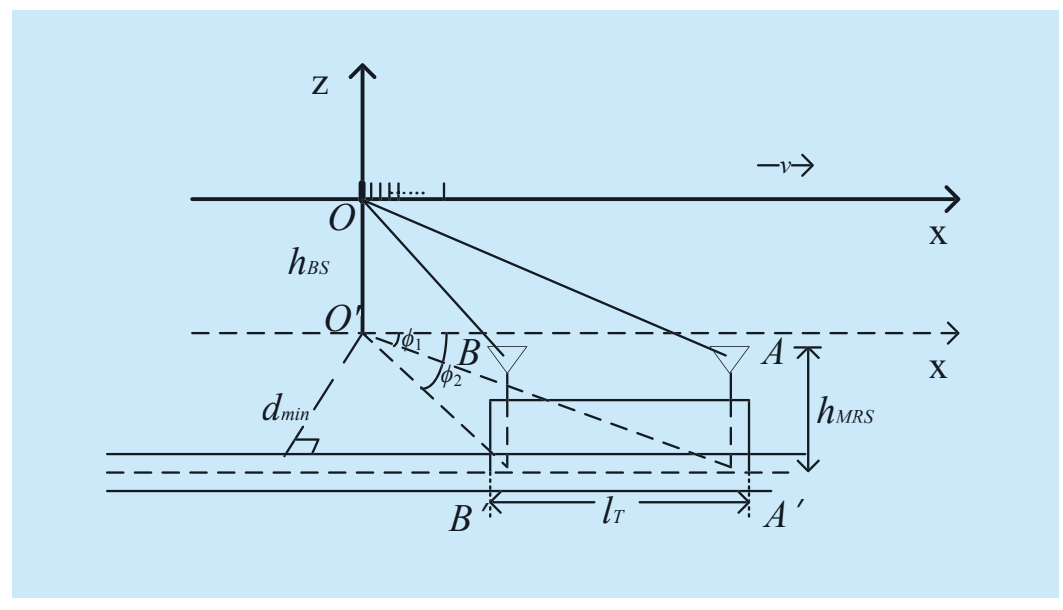

Fig.1 Passenger-oriented communication system for HSR 
al-beam beamforming technique in HSR scenarios for two main reasons. Firstly, most of the HSR environments have a strong LOS path in open rural areas, which results in a high correlation amongst the channels. Secondly, when using a small number of BS AEs, we have a low angular resolution, which further aggravates the IBA since the difference between $\phi_{1}$ and $\phi_{2}$ in Fig.1 becomes small when the train is approaching the cell edge. Thus, dual-beam beamforming can only be effective in a limited area (i.e. around the cell center). By contrast, when relying on pencil beams generated by LS-MIMO arrays, the effective serving area of dual-beam beamforming is significantly extended. Let $x_{1}$ and $x_{2}$ denote the transmitted signals targeting at the MRS antennas $A$ and $B$, respectively, assuming that $\mathrm{E}\left\{\left|x_{i}\right|^{2}\right\}=1, i=1,2$. Without loss of generality, the received signals at MRS antennas are given by:

$\left\{\begin{array}{l}y_{1}=\sqrt{\beta_{1} \frac{\rho_{f}}{N_{B}}} h_{1}^{H} w_{1} x_{1}+\sqrt{\beta_{2} \frac{\rho_{f}}{N_{B}}} h_{1}^{H} w_{2} x_{2}+n_{1} \\ y_{2}=\sqrt{\beta_{2} \frac{\rho_{f}}{N_{B}}} h_{2}^{H} w_{2} x_{2}+\sqrt{\beta_{1} \frac{\rho_{f}}{N_{B}}} h_{2}^{H} w_{1} x_{1}+n_{2}\end{array}\right.$

where $\rho_{\mathrm{f}}, N_{\mathrm{B}}, \beta_{\mathrm{i}}, \boldsymbol{h}_{\mathrm{i}}, \boldsymbol{w}_{\mathrm{i}}$ and $n_{\mathrm{i}}$ represent the total transmitting power, the number of serving beams, the composite effect of the pathloss and shadow fading from the BS to MRS antenna $i$, the channel vectors, the beamforming weight vectors and additive white Gaussian noise (AWGN) imposed on the spatial streams, respectively, and we have $n_{\mathrm{i}} \sim N(0,1)$, $i=1,2$. With the aid of the offline relative calibration technique that is used in the Argos prototype [28], the channel reciprocity relying on time-division duplexing (TDD) can be used to obtain near-perfect channel estimation from limited number of pilot sequences.

To be more specific, we have $h_{\mathrm{i}}=\alpha_{\mathrm{i}} e^{\lambda} f\left(\phi_{\mathrm{i}}\right)$, $i=1,2$, where $i=\sqrt{-1}, \alpha_{\mathrm{i}} \sim N(0,1), s_{\mathrm{i}}$ denotes the distance between the reference $\mathrm{AE}$ of the BS and the MRS antenna $i$ (i.e. $O^{\prime} A^{\prime}$ or $O^{\prime} B^{\prime}$ in Fig. 1) and $f\left({ }^{*}\right)$ is the array steering vector. There are many ways to calculate beamforming weight vector $w_{\mathrm{i}}$. The DOA based method [29] is used:

$$
\begin{aligned}
w_{i} & =\frac{1}{\sqrt{N}} f\left(\phi_{i}\right) \\
& =\frac{1}{\sqrt{N}}\left[\begin{array}{llll}
1 & e^{-j 2 \pi \Delta \cos \phi_{i}} & \cdots & e^{-j 2 \pi(N-1) \Delta \cos \phi_{i}}
\end{array}\right]^{T .}
\end{aligned}
$$

Thus the received SINR at Antenna $A$ is expressed as (5). In this formula, the derivation from $(5 b)$ to $(5 c)$ is due to the fact that $\mathrm{E}\left\{\left|x_{i}\right|^{2}\right\}=1$ and $n_{\mathrm{i}} \sim N(0,1)$. When $\alpha_{i} e^{\frac{j 2 \pi s_{i}}{\lambda}} f\left(\phi_{i}\right)$ and $\frac{1}{\sqrt{N}} f\left(\phi_{i}\right)$ are put into (5c) instead of $h_{\mathrm{i}}$ and $w_{\mathrm{i}},(5 \mathrm{~d})$ is obtained. (5e) is got as a result of $\left|f^{\mathrm{H}}\left(\phi_{1}\right) f^{\mathrm{H}}\left(\phi_{2}\right)\right|=N$ from (5d). When $N$ approaches infinity, for $\phi_{1} \neq \phi_{2}$, we have (6).

$$
\begin{aligned}
& \Gamma_{1}=\frac{\mathrm{E}\left\{\left|\sqrt{\beta_{1} \frac{\rho_{f}}{N_{B}}} h_{1}^{H} w_{1} x_{1}\right|^{2}\right\}}{\mathrm{E}\left\{\left|n_{1}+\sqrt{\beta_{2} \frac{\rho_{f}}{N_{B}}} h_{1}^{H} w_{2} x_{2}\right|^{2}\right\}} \\
& =\frac{\mathrm{E}\left\{\left|x_{1}\right|^{2}\right\}\left|\sqrt{\beta_{1} \frac{\rho_{f}}{N_{B}}} h_{1}^{H} w_{1}\right|^{2}}{\mathrm{E}\left\{\left|n_{1}\right|^{2}\right\}+\mathrm{E}\left\{\left|x_{2}\right|^{2}\right\}\left|\sqrt{\beta_{2} \frac{\rho_{f}}{N_{B}}} h_{1}^{H} w_{2}\right|^{2}}(5 b) \\
& \leqslant \frac{\left|\sqrt{\beta_{1} \frac{\rho_{f}}{N_{B}}} h_{1}^{H} w_{1}\right|^{2}}{\left|\sqrt{\beta_{2} \frac{\rho_{f}}{N_{B}}} h_{1}^{H} w_{2}\right|^{2}} \\
& \approx \frac{\beta_{1}}{\beta_{2}} \frac{\left|f^{H}\left(\phi_{1}\right) f\left(\phi_{1}\right)\right|^{2}}{\left|f^{H}\left(\phi_{1}\right) f\left(\phi_{2}\right)\right|^{2}} \\
& =\frac{\beta_{1}}{\beta_{2}} \frac{1}{\left|\frac{1}{N} f^{H}\left(\phi_{1}\right) f\left(\phi_{2}\right)\right|^{2}} \\
& =\frac{\beta_{1}}{\beta_{2}} \frac{1}{\gamma^{2}} \\
& \gamma=\frac{1}{N}\left|f^{H}\left(\phi_{1}\right) f\left(\phi_{2}\right)\right| \\
& =\frac{1}{N}\left|\sum_{n=0}^{N-1} e^{j 2 \pi \Delta\left(\cos \phi_{1}-\cos \phi_{2}\right)}\right| \\
& =\frac{1}{N}\left|\frac{1-e^{j 2 \pi \Delta\left(\cos \phi_{1}-\cos \phi_{2} N N\right.}}{1-e^{j 2 \pi \Delta\left(\cos \phi_{1}-\cos \phi_{2}\right)}}\right| \stackrel{N \rightarrow \infty}{\longrightarrow} 0
\end{aligned}
$$

As shown in Fig. 2(a), when the distance between the BS and the MRS is $500 \mathrm{~m}$, an obvious periodic change of $\gamma^{2}$ against $N$ is observed, e.g. when $N$ is increased from around 240 to 340 , instead of decreasing, $\gamma^{2}$ increases as well, as indicated by the arrow. This implies that $\gamma$ is not a monotonically decreasing function of $N$. In a comparison, Fig. 2(b) illustrates how the IBA, which is defined as the normalized value of $\left|f^{\mathrm{H}}\left(\phi_{1}\right) f^{\mathrm{H}}\left(\phi_{2}\right)\right|^{2}$, changes with locations for fixed value of $N=260$.

In Fig. 2, it is assumed that $d_{\min }$ equals $50 \mathrm{~m}$ 
while $R$ equals $1000 \mathrm{~m}$ and $s$ changes from $d_{\min }$ to $R$ when the train moves from the BS to the cell edge. When $s$ is larger than a certain value, the IBA starts to increase rapidly. From then on, dual-beam beamforming will be ineffective.

\subsection{Adaptive antenna-activation (AAA) based beamforming scheme of LS-MIMO}

In order to improve the entire transmission performance while the train moves through the cell, we propose an AAA based beamforming scheme taking advantage of LS-MIMO. In the area close to BS, all the BS AEs are activated to generate two beams of highest resolution. As the IBA starts to experience obvious ups and downs, that is, when the distance $s$ between the BS and the MRS is bigger than $s_{1}$, only $N_{\min }$ AEs of the BS are activated to satisfy the angular resolution requirement, since from then on the IBA is no longer a monotonically decreasing function of $N$. The proposed AAA scheme intends to achieve a tradeoff between high array gain and low IBA. When the IBA starts to increase monotonically, that is, when $s$ is bigger than $s_{2}$, dual-beam beamforming is no longer employed and the array switches to the single-beam beamforming with highest resolution. Assuming that the train goes through the whole cell at a constant speed, the instantaneous SE of different districts can be expressed as:

$\overline{\mathfrak{R}}=\left\{\begin{array}{lc}\sum_{i=1}^{2} \log \left(1+\Gamma_{i}\right), & d_{\min } \leqslant s<s_{1} ; \\ \sum_{i=1}^{2} \log \left(1+\widetilde{\Gamma}_{i}\right), & s_{1} \leqslant s<s_{2} ;(7) \\ \log \left(1+\beta \rho_{f}\left|h^{H} w\right|_{2}^{2}\right), & s_{2} \leqslant s \leqslant R ;\end{array}\right.$

where $\Gamma_{\mathrm{i}}$ refers to the instantaneous received SINR of Antenna $i$ when BS generates beams with all the AEs activated, while $\Gamma_{\mathrm{i}}$ denotes the instantaneous received SINR corresponding to the case where BS activates $N_{\min }$ AEs to generate beams. Additionally, $\beta, \boldsymbol{h}$ and $\boldsymbol{w}$ refer to the normalized pathloss and shadow fading from the BS to the MRS antenna, the channel vector and the beamforming weight vecto, respectively, when the single-beam beamforming is

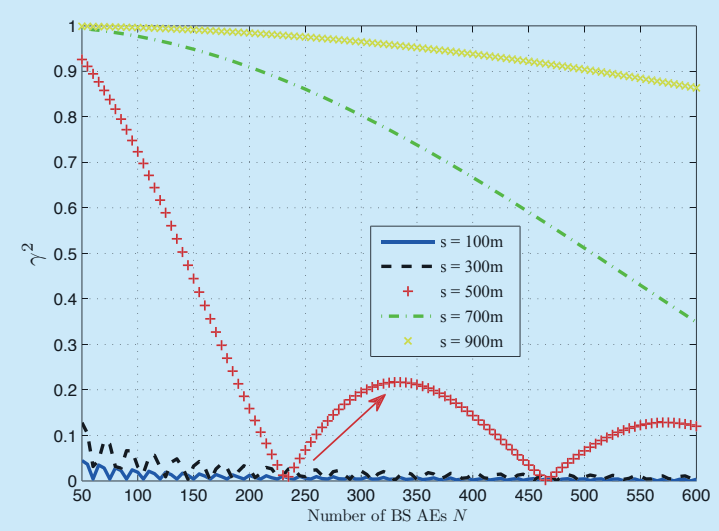

(a)

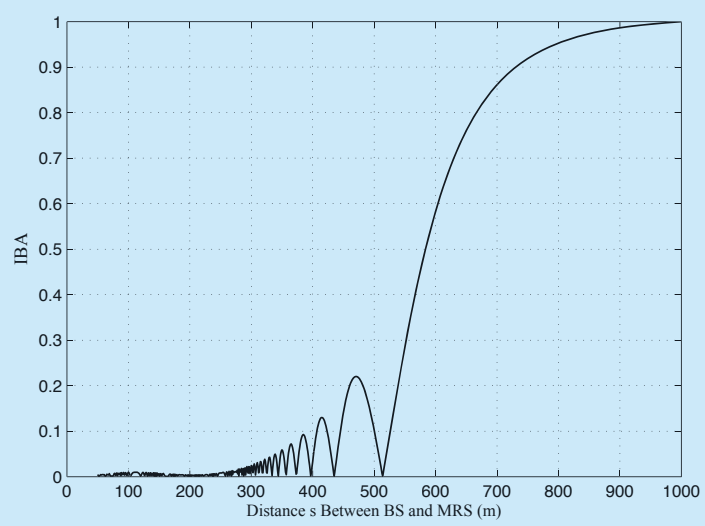

(b)

Fig.2 (a) $\gamma^{2}$ at different locations with the number of active BS AEs N; (b) IBA with fixed $N=260$ and varying locations

employed.

\section{Performance Evaluation}

In LS-MIMO communication systems of HSR, the beamforming technique is mainly used to improve the throughput of downlink transmission. In this section, the proposed AAA based beamforming and some other benchmark beamforming schemes are analyzed and compared through analytical and simulation results.

\subsection{Analytical results}

For the proposed scheme, $s_{1}$ and $s_{2}$ are the crucial parameters representing the adaptation 
threshold, the values of which are decided by the IBA. As seen in Fig.2, the IBA of $N_{\max }$ active BS AEs aided dual-beam beamforming scheme approaches zero in the area near to the BS. As the train moves away from the cell center to the cell edge, it suffers ups and downs and then turns to be intolerably high. This can be considered as the cost to maintain doubled data rate due to dual beams. Fig. 3 compares the IBA in $N_{\max }$-dual-beam and $N_{\min }{ }^{-}$ dual-beam beamforming schemes, in which the dashed line refers to the former and the solid one denotes the latter. We can see that after the point of $s_{2}$, the dashed and solid lines become identical since according to (3) $N_{\min }$ has gradually increased to $N_{\max }$ from then on. By comparison, it is shown that the IBA of the $N_{\max }$-dual-beam beamforming scheme is lower than 0.08 when $N_{\text {min }}<<N_{\max }$, which corresponds to the district before $s_{1}$. Hence, $s_{1}$ and $s_{2}$ divide $\left[d_{\min }, R\right]$ into 3 districts.

District 1: $s \in\left[d_{\min }, s_{1}\right)$

In this district, the distance between the antenna on the front of the train and the BS increases from $d_{\min }$ to $s_{1}$ while the distance between the BS and the antenna on the tail of the train firstly reduces and then increases. As this district is very close to the $\mathrm{BS}$, the difference between two directional cosines (i.e. $\cos \left(\phi_{1}\right)$ and $\left.\cos \left(\phi_{2}\right)\right)$ is very obvious, thus the value

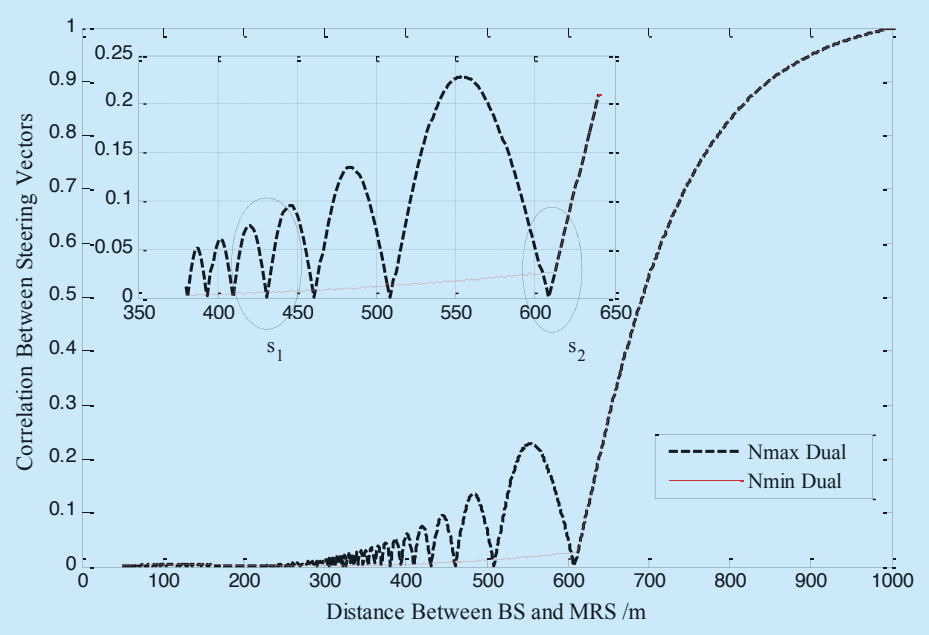

Fig.3 The IBAs of $N_{\max }$-Dual-beam and $N_{\min }$-Dual-beam beamforming schemes of $N_{\min }$ calculated from (3) is relatively small. The $N_{\text {max }}$-dual-beam beamforming scheme benefits from the full BS antenna array and two orthogonal spatial streams in this district while $N_{\min }$-dual-beam beamforming scheme has smaller array gain. Hence, in District 1, the optimal scheme is $N_{\max }$-dual-beam beamforming scheme and its achievable SE is characterized as:

$$
\begin{aligned}
& \bar{C}_{N_{\max }-\text { Dual }}=\mathrm{E}_{\alpha_{1}, \phi_{i}}\left\{\sum_{i=1}^{2} \log _{2}\left(1+\Gamma_{i}\right)\right\} \\
& \quad=\log _{2}\left(1+\mathrm{E}_{\alpha_{1}, \phi_{1}}\left\{\Gamma_{1}\right\}\right)+\log _{2}\left(1+\mathrm{E}_{\alpha_{2}, \phi_{2}}\left\{\Gamma_{2}\right\}\right) \\
& \quad \leqslant \log _{2}\left(1+\mathrm{E}_{\phi_{1}}\left\{\frac{\beta_{1}}{\beta_{2}} \frac{\left|N_{\max }\right|^{2}}{\eta_{N_{\max }}\left(\cos \phi_{1}-\cos \phi_{2}\right)}\right\}\right) \\
& \quad+\log _{2}\left(1+\mathrm{E}_{\phi_{2}}\left\{\frac{\beta_{2}}{\beta_{1}} \frac{\left|N_{\max }\right|^{2}}{\eta_{N_{\max }}\left(\cos \phi_{2}-\cos \phi_{1}\right)}\right\}\right)
\end{aligned}
$$

where we define $\eta_{N_{\max }}(x)=\left|\sum_{m=1}^{N_{\text {max }}} e^{j 2 \pi \Delta(m-1) x}\right|^{2}$.

District 2: $s \in\left[s_{1}, s_{2}\right)$

In this district, the IBA of the $N_{\max }$-dualbeam beamforming scheme begins to aggravate in an obvious periodic manner and the amplitude becomes larger and larger. The increasing IBA will degrade BER and reduce the achievable SE. The $N_{\min }$-dual-beam beamforming scheme maintains very stable performance with the minimum number of active AEs to full fill the resolution requirement at the cost of array gain. Therefore, in District 2, the optimal scheme is $N_{\min }$-dual-beam beamforming scheme and its achievable SE is characterized by:

$$
\begin{aligned}
& \bar{C}_{N_{\text {min }}-\text { Dual }}=\mathrm{E}_{\alpha_{1}, \phi_{i}}\left\{\sum_{i=1}^{2} \log _{2}\left(1+\widetilde{\Gamma}_{i}\right)\right\} \\
& \quad=\log _{2}\left(1+\mathrm{E}_{\alpha_{1}, \phi_{1}}\left\{\widetilde{\Gamma}_{1}\right\}\right)+\log _{2}\left(1+\mathrm{E}_{\alpha_{2}, \phi_{2}}\left\{\widetilde{\Gamma}_{2}\right\}\right) \\
& \quad \leqslant \log _{2}\left(1+\mathrm{E}_{\phi_{1}}\left\{\frac{\beta_{1}}{\beta_{2}} \frac{\left|N_{\min }\right|^{2}}{\eta_{N_{\min }}\left(\cos \phi_{1}-\cos \phi_{2}\right)}\right\}\right) \\
& \quad+\log _{2}\left(1+\mathrm{E}_{\phi_{2}}\left\{\frac{\beta_{2}}{\beta_{1}} \frac{\left|N_{\min }\right|^{2}}{\eta_{N_{\min }}\left(\cos \phi_{2}-\cos \phi_{1}\right)}\right\}\right)
\end{aligned}
$$

where we define $\eta_{N_{\min }}(x)=\left|\sum_{m=1}^{N_{\text {min }}} e^{j 2 \pi \Delta(m-1) x}\right|^{2}$.

District 3: $s \in\left[s_{2}, R\right]$

In this district, the $N_{\min }$-dual-beam beam- 
forming scheme has identical performance to the $N_{\max }$-dual-layer beamforming scheme since all the AEs of BS have to be activated (i.e. $N_{\min }=N_{\max }$ ) for satisfying the angular resolution requirement. However, the IBA of this scenario quickly becomes intolerable with the movement of the train which results in ineffective dual-beam beamforming. At this time, if one of the spatial streams is turned off (e.g. Antenna A) and the $N_{\max }$-single-beam beamforming is carried out, then at least it will benefit from the full BS antenna array. As a result of the tradeoff between the IBA and the array gain, in District 3, the optimal scheme is the $N_{\text {max }}$-single-beam beamforming scheme and its achievable $\mathrm{SE}$ is characterized by:

$$
\begin{aligned}
\bar{C}_{N_{\max } \text { Single }}= & \mathrm{E}_{\alpha, \phi}\left\{\log _{2}(1+\Gamma)\right\} \\
& =\log _{2}\left(1+\mathrm{E}_{\alpha, \phi}\left\{\alpha^{2} \beta \rho_{f} N_{\max }\right\}\right)
\end{aligned}
$$

According to the advantages and disadvantages of the above schemes in different districts, the proposed AAA based beamforming scheme can always maintain a good and stable performance by always executing the optimal beamforming scheme in each district. Its achievable SE is characterized by:

$\bar{C}_{A A A}=\tau_{1} \bar{C}_{N_{\max } \text {-Dual }}+\tau_{2} \bar{C}_{N_{\min } \text {-Dual }}+\tau_{3} \bar{C}_{N_{\max } \text {-Single }}$,

where $\tau_{1}=\frac{\sqrt{s_{1}^{2}-d_{\min }^{2}}}{\sqrt{R^{2}-d_{\min }^{2}}}$,

$\tau_{2}=\frac{\sqrt{s_{2}^{2}-d_{\min }^{2}}-\sqrt{s_{1}^{2}-d_{\text {min }}^{2}}}{\sqrt{R^{2}-d_{\text {min }}^{2}}}$ and

$\tau_{3}=\frac{\sqrt{R^{2}-d_{\min }^{2}}-\sqrt{s_{2}^{2}-d_{\min }^{2}}}{\sqrt{R^{2}-d_{\min }^{2}}}$.

\subsection{Analysis of robustness against DOA estimation error}

In HSR scenario, the speed and location information of the train is fed back to the BS via the CBTC subsystem which facilitates the DOA estimation. When there exists some error in location prediction, the DOA estimation will be affected, which in turn affects the accuracy of the beamforming weight vector $\boldsymbol{w}_{\mathrm{i}}$. Assume the inaccuracy of DOA estimation in HSR communication system is $\xi(0 \leq \xi \leq 1)$, then the estimated DOA is $\widehat{\phi}=\phi \pm \xi \phi$, where $\phi$ is the true DOA. Since DOA is a crucial param- eter for generating steering vectors, the DOA estimation error will affect the performance of these DOA based beamforming schemes.

Let $\Gamma_{i}^{*}$ denote the SINR with DOA estimation error at the receive antenna $i$, then we have

$$
\begin{array}{r}
\Gamma_{1}^{*} \approx \frac{\beta_{1} \eta\left(\cos \phi_{1}-\cos \widehat{\phi}_{1}\right)}{\beta_{2} \eta\left(\cos \phi_{1}-\cos \widehat{\phi}_{2}\right)}, \\
\Gamma_{2}^{*} \approx \frac{\beta_{2} \eta\left(\cos \phi_{2}-\cos \widehat{\phi}_{2}\right)}{\beta_{1} \eta\left(\cos \phi_{2}-\cos \widehat{\phi}_{1}\right)} .
\end{array}
$$

The robustness against the DOA estimation error of these beamforming schemes can be evaluated by the achievable SE and the normalized SE loss. For the proposed scheme, different $\xi$ has different influence in each adaptation district. Some district is more sensitive to DOA errors as shown in the next section.

\subsection{Simulation results}

In this section, numerical simulations were conducted to evaluate the performance of the proposed scheme. $R$ and $d_{\text {min }}$ are still set as $1000 \mathrm{~m}$ and $50 \mathrm{~m} . N$ is assumed to be up to 260 and the carrier frequency is set as $3.5 \mathrm{GHz}$ [31]. Note that it is suggested that the carrier frequency of LS-MIMO might be increased to reduce the size of array [24]. As mentioned in Section III, with the internal relative calibration method [28], near-perfect channel estimation can be achieved. Hence, for simplicity, we assume perfect CSI. The speed of the train is assumed constant at $100 \mathrm{~m} / \mathrm{s}$ (i.e. $360 \mathrm{~km} / \mathrm{h}$ ) and it is simplified as moving along a straight track. With the speed and location information, it can also be assumed that the Doppler frequency offset estimation and compensation are perfect.

Fig.4 and Fig.5 illustrate the performances of various beamforming schemes considered in terms of the time-average distance-related (TADR) BER and the achievable SE, respectively. The transmit SNR, s1 and s2 are 10 $\mathrm{dB}, 440 \mathrm{~m}$ and $612 \mathrm{~m}$, respectively. In Fig.4, the Nmax-dual-beam beamforming scheme has similar BER performance which is around 


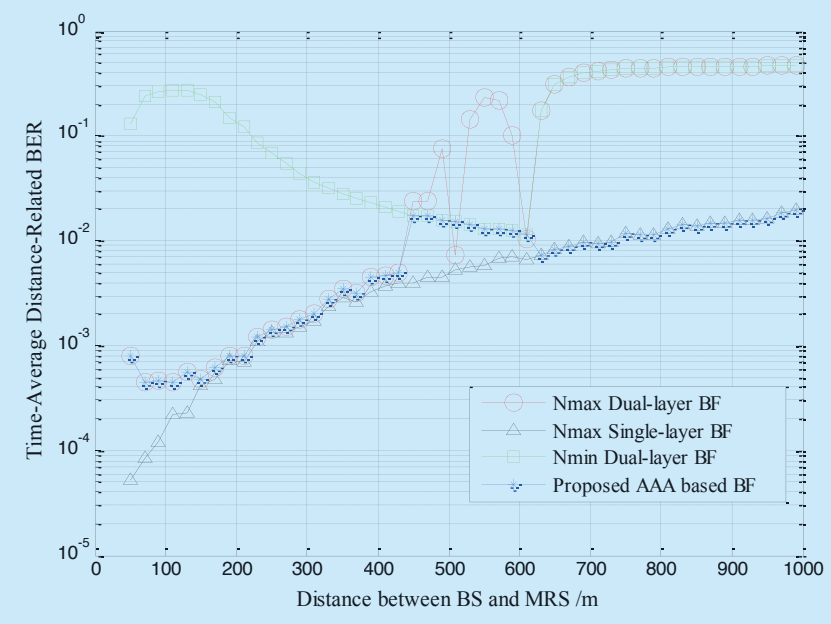

Fig.4 BER performance of beamforming schemes

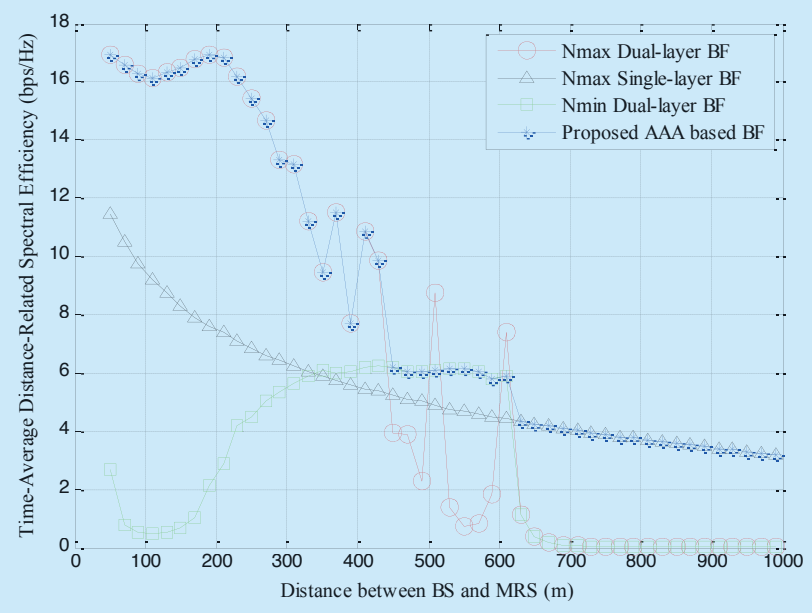

Fig.5 Spectral efficiency of beamforming schemes

10-2 as the single-beam beamforming scheme before s1 while the BER performance of the Nmin-dual-beam beamforming is higher than 10-2. In District 2, the BER of the Nmindual-beam beamforming improves steadily, but the BER of the Nmax-dual-beam beamforming starts to fluctuate and becomes even higher than 10-1. After s2, the dual-beam schemes suffer very high BER which makes the dual-beam beamforming ineffective. In
District 3, the proposed scheme employs the single-beam beamforming to reduce the BER from 10-1 to 10-2.

In Fig.5, the advantage of the $N_{\max }$-dualbeam beamforming in terms of the achievable SE is very obvious in District 1 , such as at the distance of $150 \mathrm{~m}$, the achievable $\mathrm{SE}$ of the $N_{\text {max }}$-dual-beam beamforming is twice of that of the $N_{\text {max }}$-single-beam beamforming and about eleven times of that of the $N_{\min }$-dualbeam beamforming. When the train moves to District 2, the achievable SE of the $N_{\min }$-dualbeam beamforming gradually surpasses that of $N_{\text {max }}$-single-beam scheme by more than 3bps/ $\mathrm{Hz}$ while the achievable SE of the $N_{\max }$-dualbeam beamforming aggravates rapidly and becomes the worst. After $s_{2}$, serious IBA as well as the increased path loss make the dual-beam beamforming schemes invalid but the $N_{\max }{ }^{-}$ single-beam scheme can maintain a $6.5 \mathrm{bps} /$ $\mathrm{Hz}$ achievable SE.

To verify the impact of DOA estimation error on the beamforming schemes, both the time-average distance-average (TADA) SE and the spectral efficiency loss ratio (SELR) are calculated when $\xi$ changes from 0 to 0.1 , as shown in Fig.6. In Fig.6(a), the four schemes considered all experience SE loss when $\xi$ is larger than 0. In Fig.6(b), the sensitivity to DOA errors of these schemes is demonstrated. It is obvious that the proposed scheme is more robust than the $N_{\max }$-dual-beam beamforming scheme which suffers $20 \%$ loss when the inaccuracy indicator $\xi$ is 0.1 , while the SELR of the proposed scheme is $16 \%$. For the $N_{\max }-\sin$ gle-beam beamforming scheme, its achievable SE is always higher than that of the $N_{\min }$-dualbeam scheme by $3 \mathrm{bps} / \mathrm{Hz}$ and its SELR is less than that of the $N_{\min }$-dual-beam scheme by $3 \sim 5 \%$.

Fig.7 describes the influence of different values of $\xi$ on the TADR SE of the proposed scheme. In District 1 and 2, the lower the inaccuracy $\xi$ is, the better the proposed scheme performs. In District 3, the TADR SE of the proposed scheme with different values of $\xi$ becomes almost identical, which means in this district this scheme is not very sensitive to 
DOA estimation errors.

\subsection{Impact of the number of active BS AEs}

The proposed AAA based beamforming scheme is mainly decided by two parameters: $s_{1}$ and $s_{2}$. These two values will divide $\left[d_{\min }\right.$, $R]$ into three districts: $\left[d_{\min }, s_{1}\right],\left[s_{1}, s_{2}\right]$ and $\left[s_{2}\right.$, $R]$, which means that, $s_{1}$ will trigger the proposed scheme to switch from the $N_{\max }$-dualbeam beamforming to the $N_{\min }$-dual-beam beamforming and $s_{2}$ will trigger the proposed scheme to switch from the $N_{\min }$-dual-beam beamforming to the $N_{\max }$-single-beam beamforming. Thus, $s_{2}$ can be calculated from (3) where $N_{\min }$ on the left hand is set to the same value of $N_{\max }$. When it comes to $s_{1}$, the IBA as shown in Fig. 3 should be taken into consideration. The distance at which the IBA of the $N_{\max }$-dual-beam beamforming surpasses a threshold (e.g. 0.08) for the first time will be taken as $s_{1}$. For different values of $N$, when $d_{\min }=50 \mathrm{~m}$ and $R=1000 \mathrm{~m}$, some empirical values of $\left(s_{1}, s_{2}\right)$ are shown in Table I.

In this table, it is illustrated that smaller $N$ will shrink the effective operating district of the dual-beam beamforming (i.e. $\left[d_{\text {min- }}\right.$ ,$\left.s_{2}\right]$ ), especially the operating district of the $N_{\max }$-dual-beam beamforming (i.e. $\left[d_{\min }, s_{1}\right]$ ). The principle of the proposed AAA based beamforming is to make use of the $N_{\max }$-dualbeam beamforming as much as possible since it can provide both higher array power gain and higher multiplexing gain. When the IBA starts to fluctuate seriously or even becomes intolerable, the proposed scheme will switch from the $N_{\max }$-dual-beam beamforming to other suboptimal schemes. At the same time, we also aim to reduce the operating district of single-beam beamforming which is triggered by $s_{2}$. Thus the larger the effective operating district of dual-beam beamforming is, the better the proposed scheme will perform. In the proposed scheme, we assume that the radius of the operating district of single-beam beamforming should not be longer than $500 \mathrm{~m}$ and the radius of the effective operating district of the $N_{\max }$-dual-beam beamforming should not

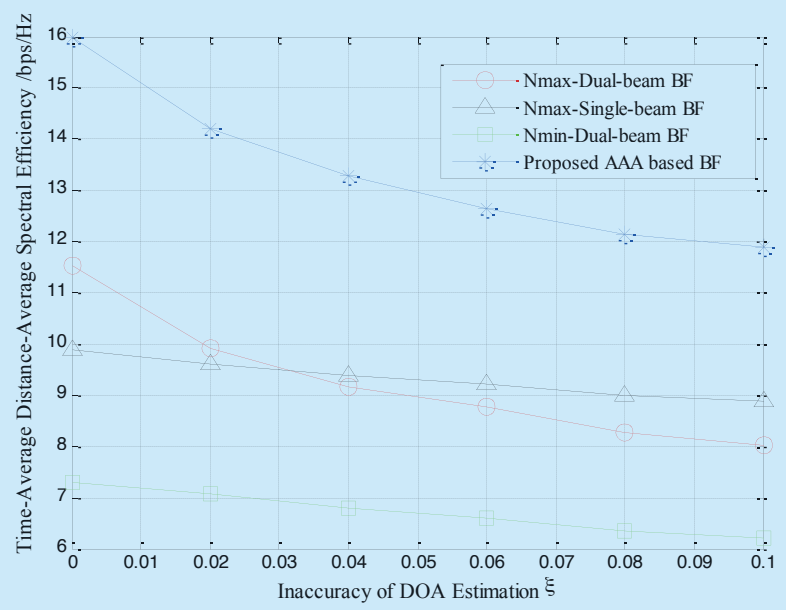

(a)

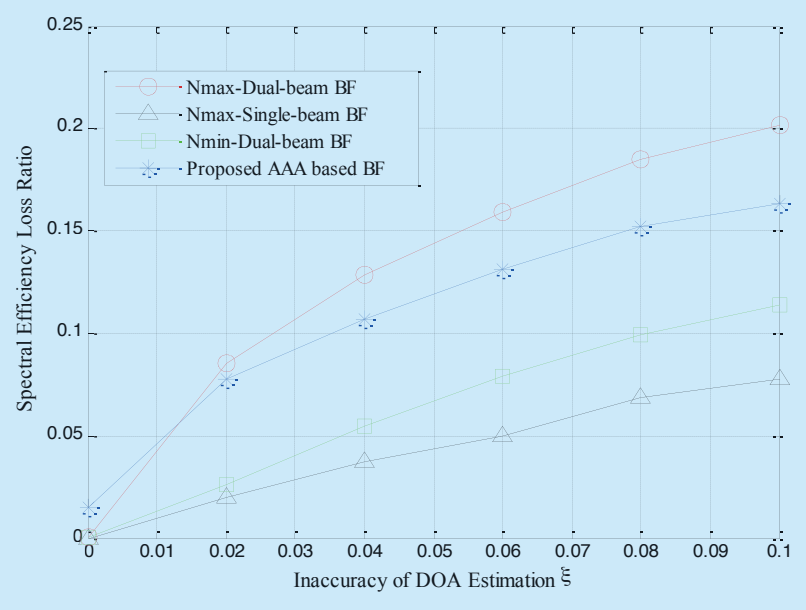

(b)

Fig.6 Robustness against DOA estimation error of different beamforming schemes: (a) TADA SE against the inaccuracy of DOA estimation; (b) SE loss ratio against the inaccuracy of DOA estimation

be shorter than $330 \mathrm{~m}$. Thus the number of BS AEs $N$ should be at least 260 which corresponds to the setting of $s_{1}=381 \mathrm{~m}, s_{2}=517 \mathrm{~m}$, the carrier frequency $3.5 \mathrm{GHz}$ and the length of the BS antenna array is $11.1 \mathrm{~m}$. In the considered configuration, when $N$ is smaller than 260 , the proposed AAA based beamforming scheme will not obtain the desired performance.

Finally, Fig. 8 shows the relationship between the TADA SE and the number of BS AEs. For all schemes, the TADA SE increas- 


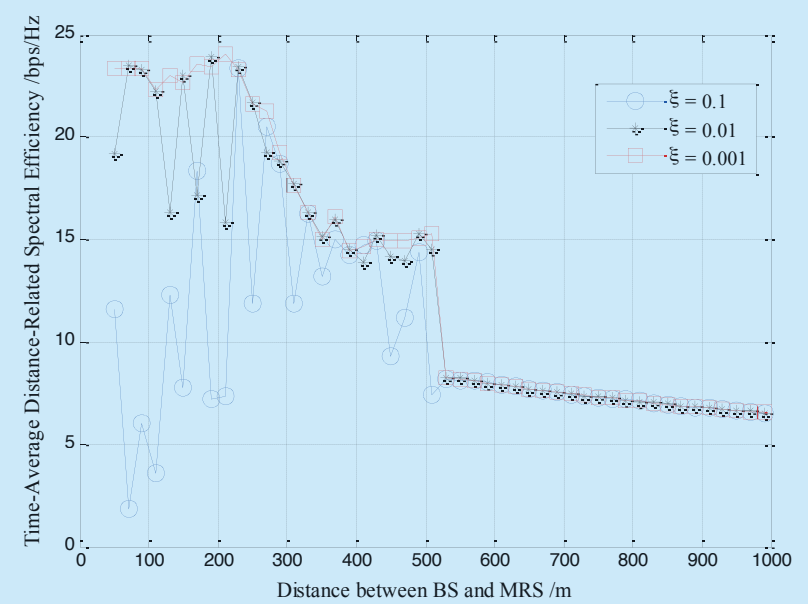

Fig.7 The TADR SE of the proposed AAA based beamforming scheme with different $\xi$

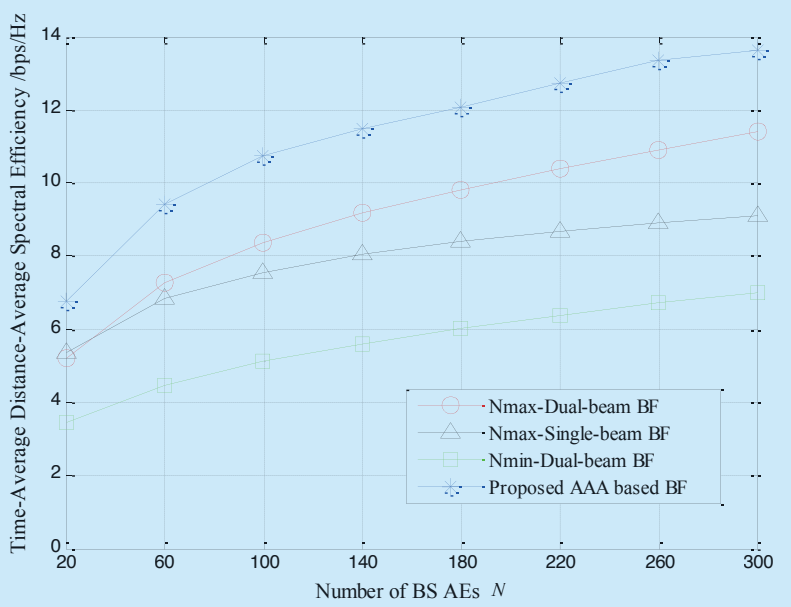

Fig.8 The TADA SE changes with the number of BS AEs

Table I Empirical values of $\left(s_{1}, s_{2}\right)$ for different values of $n$ and the IBA threshold of 0.08

\begin{tabular}{cccccc}
\hline $\mathrm{N}$ & 100 & 200 & 300 & 400 & 500 \\
$\left(\mathrm{~s}_{1}, \mathrm{~s}_{2}\right)$ & $(315,412)$ & $(320,485)$ & $(392,536)$ & $(418,578)$ & $(440,612)$ \\
\hline
\end{tabular}

es with $N$. The performance of the proposed scheme increases fastest among them. Moreover, the larger the $N$ is, the more the pro- posed scheme outperforms the $N_{\max }$-dual-beam beamforming scheme.

\section{CONCLUSION AND FutURE WORK}

This paper proposed an AAA based beamforming scheme for LS-MIMO aided communication systems of HSR scenario. To obtain both the array gain and the spatial multiplexing gain, all the AEs of the BS array are activated to form two beams serving antennas on the front and tail of the train respectively. However, it is observed that the closer the train moves to the cell edge, the higher the IBA will be. Thus, according to the IBAs of the $N_{\max }$ - and the $N_{\min }$-dual-beam beamforming schemes, the whole district is divided into three districts. The proposed scheme activates different number of BS AEs and generates distinct number of serving beams in different district. Analytical and simulation results show that the proposed scheme not only has the best performance in terms of BER and achievable $\mathrm{SE}$, but also has relatively higher robustness to DOA estimation errors. In our future work, the analytical results on the asymptotic SE of the dual-beam beamforming schemes under HSR scenario will be investigated. We will also extend the results presented in this paper to the more generalized multi-beam beamforming scenario.

\section{ACKNOWLEDGEMENT}

This study was supported partially by the 973 Program under the Grant 2012CB316100.

\section{References}

[1] T.T Gao, B Sun, "A High-Speed Railway Mobile Communications System based on LTE", Proceedings of 2010 International Conference Electronic and Information Engineering, vol.1, pp 414-417, Aug, 2010.

[2] L.H Lu, X.M Fang, M Cheng, et al., "Positioning and Relay Assisted Robust Handover Scheme for High Speed Railway", Proceedings of 2011 IEEE Vehicular Technology Conference, pp 1-5, May, 2011

[3] K Guan, Z.D Zhong, B Ai, "Assessment of LTE-R Using High Speed Railway Channel Model", Proceedings of $3^{\text {rd }}$ International Conference on 
Communications and Mobile Computing, pp 461-464, April, 2011.

[4] H.H Wang, H.A Hou, "Experimental Analysis of Beamforming in High-Speed Railway Communication", Proceedings of $22^{\text {nd }}$ International Symposium on Personal Indoor and Mobile Radio Communications, pp 745-749, Sep, 2011.

[5] B.J Han, Y.M Liang, L Huo, et al., "Coexistence of Downlink High-Speed Railway Communication System with TDD-LTE Cellular Communication System", Proceedings of 2012 IEEE Vehicular Technology Conference, pp 1-6, May, 2012.

[6] L Tian, J Li, Y Huang, et al., "Seamless Dual-Link Handover Scheme in Broadband Wireless Communication Systems for High-Speed Rail", IEEE Journal on Selected Areas in Communications, vol.30, no.4, pp 708-718, May, 2012.

[7] R.S He, Z.D Zhong, B Ai, et al., "Measurements and Analysis of Propagation Channels in HighSpeed Railway Viaducts", IEEE Transactions on Wireless Communications, vol.12, no.2, pp 794805, Dec, 2012

[8] Y Sun, C.Y Lee, J.M Jo, et al., "Study on the Effectiveness of High-Speed Railway Communication and Signaling System based on 4G LTE Technology", Proceedings of $13^{\text {th }}$ International Conference on Control, Automation and Systems, pp 402-406, Oct, 2012.

[9] W.T Luo, X.M Fang, M Cheng, et al., "Efficient Multiple-Group Multiple-Antenna (MGMA) Scheme for High-Speed Railway Viaducts", IEEE Transactions on Vehicular Technology, vol.62, no.6, pp 2558-2569, Feb, 2013.

[10] M Cheng, X.M Fang, W.T Luo, "Beamforming and Positioning-Assisted Handover Scheme for Long-Term Evolution System in High-Speed Railway", IET Communications, vol.6, no.15, pp 2335-2340, Oct, 2012.

[11] M Cheng, X.M Fang, "Location Information-Assisted Opportunistic Beamforming in LTE System for High-Speed Railway", Eurasip Journal on Wireless Communications and Networking, vol.1, pp 1-7, July, 2012.

[12] M Cheng, X.M Fang, L Yan, "Beamforming and Alamouti STBC Combined Downlink Transmission Schemes in Communication Systems for High-Speed Railway", Proceedings of International Conference on Wireless Communications and Signal Processing, pp 1-6, Oct, 2013.

[13] R.D Pascoe, T.N Eichorn, "What Is Communication-Based Train Control?", IEEE Vehicular Technology Magazine, vol.4, no.4, pp 16-21, Dec, 2009.

[14] D Tse, P Viswanath, "Fundamentals of Wireless Communication", Cambridge University Press, 2005.

[15] T.L Marzetta, "Noncooperative Cellular Wireless With Unlimited Numbers of Base Station Antennas", IEEE Transactions on Wireless Communications, vol.9, no.11, pp 3590-3600, Nov, 2010.
[16] F Rusek, D Persson, B.K Lau, et al., "Scaling up MIMO: Opportunities and Challenges with Very Large Arrays", IEEE Signal Processing Magazine, vol.30, no.1, pp 40-60, Jan, 2013.

[17] E.P Tsakalaki, O.N Alrabadi, E.D Carvalho, et al., "On the Beamforming Performance of LargeScale Antenna Arrays", Proceedings of 2012 Loughborough Antennas and Propagation Conference, pp 1-4, Nov, 2012.

[18] J Hoydis, B.S Ten, M Debbah, "Comparison of Linear Precoding Schemes for Downlink Massive MIMO", Proceedings of 2012 IEEE International Conference on Communications, pp 21352139, June, 2012.

[19] X Gao, O Edfors, F Rusek, et al., "Linear Pre-Coding Performance in Measured VeryLarge MIMO Channels", Proceedings of 2011 IEEE Vehicular Technology Conference, pp 1-5, Sep, 2011.

[20] J Jose, A Ashikhmin, T.L Marzetta, et al., "Pilot Contamination and Precoding in Multi-Cell TDD Systems", IEEE Transactions on Wireless Communications, vol.10, no.8, pp 2640-2651, Aug, 2011.

[21] A Shikhmin, T.L Marzetta, "Pilot Contamination Precoding in Multi-Cell Large Scale Antenna Systems", Proceedings of 2012 IEEE International Symposium on Information Theory, pp 11371141, July 2012.

[22] J Hoydis, B.S Ten, M Debbah, "Massive MIMO in the UL/DL of Cellular Networks: How Many Antennas Do We Need?", IEEE Journal on Selected Areas in Communications, vol.31, no.2, pp 160171, Feb, 2013.

[23] O.N Alrabadi, H Tsakalaki, H Huang, et al., "Beamforming via Large and Dense Antenna Arrays above a Clutter", IEEE Journal on Selected Areas in Communications, vol.31, no.2, pp 314325, Feb, 2013.

[24] Z.Y Pi, "Optimal Transmitter Beamforming with Per-Antenna Power Constraints", Proceedings of 2012 IEEE International Conference on Communications, pp 3779-3784, June, 2012.

[25] H Huh, A Tulino, G Caire, "Network-MIMO Cellular Systems: Large-System Analysis and Low-Overhead Downlink Scheduling", Proceedings of 2011 IEEE Statistical Signal Processing Workshop, pp 693-696, June, 2011.

[26] H Huh, G Caire, H.C Papadopoulos, et al., "Achieving Large Spectral Efficiency with TDD and Not-So-Many Base-Station Antennas", Proceedings of 2011 IEEE-APS Topical Conference on Antennas and Propagation in Wireless Communications, pp 1346-1349, Sep, 2011.

[27] T.L Marzetta, "How Much Training is Required for Multiuser MIMO?", Proceedings of $40^{\text {th }}$ Asilomar Conference on Signals, Systems and Computers, pp 359-363, Oct, 2006.

[28] C Shepard, H Yu, N Anand, et al., "Argos: Practical Many-Antenna Base Stations", Proceedings 
of $18^{\text {th }}$ Annual International Conference on Mobile Computing and Networking, pp 53-64, Aug, 2012.

[29] C.A Balanis, "Antenna Theory: Analysis and Design", John Wiley \&Sons Ltd., 2012.

[30] H.Q Ngo, T.L Marzetta, E.G Larsson, "Analysis of the Pilot Contamination Effect in Very Large Multicell Multiuser MIMO Systems for Physical Channel Models", Proceedings of 2011 IEEE International Conference on Acoustics, Speech and Signal Processing, pp 3464-3467, May, 2011.

[31] S Sesia, I Toufik, M Baker, "LTE: the UMTS Long Term Evolution", John Wiley \&Sons Ltd., 2011.

\section{Biographies}

Meng Cheng, received the B.E. degree in Information Security in 2008, the Ph.D. degree in Communications and Information System in 2015 from Southwest Jiaotong University, Chengdu, China. She was funded by China Scholarship Council as a joint-training Ph.D. to study in University of Southampton, United Kingdom from October 2012 to April 2014. She is currently doing her post-doctoral research in Wuhan Railway Bureau. Her research interests include wireless communications for high-speed railway, prog- nostic and health management.

Shaoshi Yang, received the B.E. degree in Information Engineering from BUPT, China and Ph.D. in Electronics and Electrical Engineering from University of Southampton, UK, where he is now a Research Fellow. He has published 30+ IEEE journal papers on MIMO signal processing, green radio, convex/ multi-objective optimization and their applications. He serves as a TPC member of major IEEE conferences and a Guest Associate Editor of IEEE Journal on Selected Areas in Communications.

Xuming Fang, received the B.E. degree in electrical engineering in 1984, the M.E. degree in computer engineering in 1989, and Ph.D. degree in communication engineering in 1999 from Southwest Jiaotong University, Chengdu, China. He has, to his credit, around 200 high-quality research papers in journals and conference publications. He has authored or co-authored five books or textbooks. His research interests include wireless broadband wireless networks, multi-hop networks, broadband wireless access for high speed railway, etc. *The corresponding author, Email: xmfang@swjtu.edu.cn 